\title{
3 Research Square

\section{Concentrated Growth Factors in Combination with Particulate Bio-Oss Graft: A Pilot Study on Beagle Dog Alveolar Bone Deficient}

\section{Fei Chen}

The First Medical Center, Chinese PLA General Hospital

Qiang Luo

The First Medical Center, Chinese PLA General Hospital

\section{Lin Wang}

The First Medical Center, Chinese PLA General Hospital

\section{Shao-xia Pan}

Peking University School of Stomatology

\section{Peng Xue ( 690940250@qq.com )}

The First Medical Center of Chinese PLA General Hospital https://orcid.org/0000-0002-8429-416X

\section{Research}

Keywords: concentrated growth factors (CGF), TGF- $\beta 1$, VEGF, integrated optical density (IOD), bone formation, mineral apposition rate (MAR).

Posted Date: November 10th, 2021

DOI: https://doi.org/10.21203/rs.3.rs-1033941/v1

License: (c) (1) This work is licensed under a Creative Commons Attribution 4.0 International License. Read Full License 


\title{
Concentrated growth factors in combination with particulate Bio-Oss
}

\section{graft: a pilot study on beagle dog alveolar bone deficient}

Fei Chen ${ }^{1 \#}$, Qiang Luo ${ }^{1 \#}$, Lin Wang ${ }^{1}$, Shao-xia Pan $^{2 *}$, Peng Xue ${ }^{1 *}$

${ }^{1}$ Institute of Stomatology, The First Medical Center, Chinese PLA General Hospital, No.28 Fuxing Road, Haidian District, Beijing 100853, China

${ }^{2}$ Department of Prosthodontics, Peking University School and Hospital of Stomatology \& National Engineering Laboratory for Digital and Material Technology of Stomatology \& Beijing Key Laboratory of Digital Stomatology, No. 22 Zhongguancun Avenue South, Haidian District, Beijing 100081, China

\#These authors contributed equally to this work

*Correspondence should be addressed to Peng Xue; 690940250@qq.com and Shao-xia Pan; sx_pan@hotmail.com

\begin{abstract}
Background: Alveolar bone related defect has emerged as a major challenge for clinician. Previous studies reported that concentrated growth factors (CGF), an autogenous product derived from venous blood, could enhance healing of grafts via releasing various growth factors.

Methods: This study was designed to investigate the osteogenesis of CGF gel accompanied with Bio-Oss in canine alveolar defect socket model. CGF gel were prepared via variable speed centrifugation. Immunohistochemical staining and semi-quantitative analysis were performed to evaluate the content of transforming growth factor- $\beta 1$ (TGF- $\beta 1$ ) and vascular endothelial growth factor (VEGF) in the CGF gel and red blood cell (RBC) layer. The decay rate of TGF- $\beta 1$ and VEGF were determined by ELISA assay. Next, mixture of Bio-Oss and CGF gel was implanted as graft to the alveolar defect socket. Three different fluorescent dyes and toluidine blue staining were utilized to track osteogenic progress, and mineral apposition rate (MAR) was calculated.
\end{abstract}


Results: In the CGF gel, TGF- $\beta 1$ occupied the dominant content with a higher decay rate than that of VEGF. Whereas, the concentration of TGF- $\beta 1$ released from CGF gel still raised up rapidly within 14 days. In the animal study, combination of Bio-Oss and CGF gel not only accelerated wound healing, but also succussed to activate and sustain the bone formation at defect site represented as a higher MAR $(2.21 \pm 0.52$ $\mu \mathrm{m} /$ day vs $1.43 \pm 0.41 \mu \mathrm{m} /$ day, Bio-Oss group and $1.04 \pm 0.26 \mu \mathrm{m} /$ day, control groups, $\mathrm{P}<0.05)$.

Conclusions: combination of Bio-Oss and CGF gel could promote osteogenesis and might provide a promising strategy against alveolar bone defect.

Keywords: concentrated growth factors (CGF); TGF- $\beta 1$; VEGF; integrated optical density (IOD); bone formation; mineral apposition rate (MAR).

\section{Background}

Rebuilding deficient alveolar bone to assist in reconstruction for dental implants has emerged as a tough task for the oral and maxillofacial surgeon. Various biomaterials have been applied clinically and experimentally, of which, autologous bone grafting was considered as the gold standard for bone reconstruction accompanied by shortcomings including limited available autologous bone, graft resorption and donor site morbidity[1, 2]. Furthermore, the acquisition of autologous bone might augment the burden on patients suffering life threatening to varying degrees, and elevate the incidence of postoperative complications. Essentially, the goal of bone defect repairing was to induce regeneration of bone tissues and promote differentiation of osteoblasts rather than ostensible defect filling, therefore, the whole process was extremely time-consuming for xenograft[3]. To overcome this inadequacy, numerous studies have been put forward to synthesize multi-functional grafts with complementary advantages via the combination of materials of different characteristics. Among these bone substitutes, deproteinized bovine bone mineral (Bio-Oss, Geistlich Pharma AG, Wolhusen, Switzerland) has been demonstrated as a 
promising material due to the formation of new bone, and increase in the width of the alveolar process, and a high implant survival rate[4]. However, recent studies revealed that the amount of newly formed bone varied between $31 \%$ and $42 \%$ with application of Bios-Oss alone, suggesting that combination of Bios-Oss and growth factors might be a potential strategy to alter the way in which maxillofacial surgeons manage bone $\operatorname{defect}[5]$.

Physiologically, bone regeneration was a complex process regulated by multiple osteo-inductive cytokines involved in inflammation, angiogenesis, and hemostasis, which were embedded within the extracellular matrix, from which they were released once trauma or remodeling occurred[6, 7]. These cytokines participated in the formation of bone via regulating cell migration, proliferation, and differentiation [8, 9]. However, due to the complexity and precision, the interactions and networks between endogenous growth factors couldn't be simulated under the available technical conditions[10]. Interestingly, most endogenous growth factors, including TGF- $\beta 1$, VEGF, insulin-like growth factor (IGF), were enriched in blood components, especially the platelets[11, 12]. Additionally, platelet gels derived from autologous blood were characterized by economic property and excellent biocompatibility that have made them widely applied in tissue healing and regenerative procedures[13].

Clinically, the techniques to concentrate platelets have been updated to the 3rd generation, and numerous biomaterials containing platelets concentrate, growth factors, and fibrin have been introduced for bone restoration[14]. Initially, platelets rich plasma (PRP), the first generation of platelet gels, was criticized for the addition of thrombin and anticoagulant and a demanding centrifugation technique for preparation. Subsequently, Choukroun et al. developed a modified form of platelet gel, the platelet-rich fibrin (PRF), that possessed a continual and steady release profile of growth factors over a 10-day period[15]. Remarkably, PRF could be obtained in a simpler method. However, the species of growth factors in PRF were relatively simple 
due to the single centrifugal force. To overcome these deficiencies, Sacco et al. introduced a novel platelet gel product, concentrated growth factors (CGF), through centrifuging blood sample at alternating speeds, which endowed CGF a large and dense fibrin matrix with massive and diverse growth factors[16]. In that way, blood could be separated to three layers including serum, CGF and red blood cell (RBC) layer. Previous studies have clarified the characteristics of CGF in vivo and in vitro[1, $10,17]$. Whereas, research on the RBC layers was still lack. Moreover, the releasing profiles of growth factor derived from CGF gel and RBC layer have not been elucidated well.

In the present study, human blood samples were subjected to a variable-speed centrifugation for analysis. The comparison between CGF gel and RBC layer on growth factors release were made. Moreover, we have tested the effect of mixture of CGF gel and Bio-Oss on osteogenesis in an alveolar defect socket model.

\section{Methods}

\section{Preparation and homogenization of CGF}

Venous blood samples were collected from six healthy volunteers (3 males and 3 females with an average age of 25.2 years) under the condition of empty stomach in the morning and stored in sterile Vacuette tubes (Greiner Bio-One, GmbH, Kremsmunster, Austria) without anticoagulant solutions. All volunteers recruited in this study were informed of the nature of this study and signed an informed consent prior to their inclusion. This study protocol was reviewed and approved by the ethical board of Peking University School of Stomatology (PKUSSIRB-201519008). Patients with hematopathy, systemic diseases, medical history of drug known to affect the number or function of platelets in the past three months, and abnormal platelet counts were excluded.

As previous described[18], $9 \mathrm{ml}$ fresh blood samples were centrifuged (Medifuge ${ }^{\mathrm{TM}}$ 
MF200, Silfradentsrl, S. Sofia Italy) using a customized program as followed: $30 \mathrm{sec}$ acceleration, $2 \mathrm{~min}$ at $2700 \mathrm{rpm}, 4 \mathrm{~min}$ at $2400 \mathrm{rpm}, 4 \mathrm{~min}$ at $2700 \mathrm{rpm}, 3 \mathrm{~min}$ at 3000 rpm, and $36 \mathrm{sec}$ deceleration and stop. Eventually, three distinct layers were accessed and respectively represented as the serum, CGF gel and RBC layer from the top to the bottom.

\section{Immunohistochemical staining}

Followed by centrifugation, blood fractions were respectively incubated at room temperature for $0 \mathrm{~min}$ (onset), $15 \mathrm{~min}, 30 \mathrm{~min}, 60 \mathrm{~min}$ and $120 \mathrm{~min}$, and then embedded in paraffin to produce sections. After dewaxing to water, antigen retrieval and endogenous peroxidase deactivation, the sections were further blocked with $5 \%$ bovine serum albumin for $1 \mathrm{~h}$ and incubated with primary antibody against TGF- $\beta 1$ and VEGF overnight at $4{ }^{\circ} \mathrm{C}$. After washing with Phosphate Buffered Saline (PBS), HRP-conjugated secondary antibody (Polink-2 plus®, Golden Bridge International, Inc.) and diaminobenzidine (ZSGB-BIO, Beijing, China) were applied for color reaction. Images were acquired via microscope.

\section{ELISA assays}

After centrifugation, RBC layer was discarded from the blood fractions, and the left superior serum and CGF gel were preserved at $37{ }^{\circ} \mathrm{C}$ together. To determine the release of growth factors originated from CGF gel, the concentrations of TGF- $\beta 1$ and VEGF in superior serum were assessed respectively at 0 (onset), 7, 14, 28 day by using ELISA kits (BlueGene Biotech, Shanghai, China) according to the manufacturer's protocol. Absorbance was measured at $450 \mathrm{~nm}$. The resulting concentrations were adjusted to final concentrations according to the dilution folds.

\section{Surgical design}

Male beagle dogs, aged 15-24 months, weighing approximately $15 \mathrm{~kg}$ were recruited in this study. Each dog occupied separate room, and was fed a standard pellet diet 
with ad libitum access to water. All dogs were bred exclusively for research purposes and had intact dentition with a healthy periodontium. All protocols were approved by the Biomedical Ethics Committee of Peking University (LA2011-060). Dogs were anesthetized with intramuscular administration of $6 \%$ pentobarbital sodium $(30 \mathrm{mg} / \mathrm{kg})$ in supplement with local infiltration anesthesia of $2 \%$ lidocaine. Venous blood was collected to obtain CGF gel as previously described. Then, the bilateral maxillary first, second, and third premolars were extracted to established a tooth-deficient socket model. The second and third extraction socket at right side (the experimental site) were grafted with mixture of CGF and Bio-Oss Collagen. Instead, the contralateral second and third extraction sockets were filled with Bio-Oss Collagen (the positive control). Finally, the left bilateral first extraction sockets were backfilled with blood clot only (the negative control). All sockets were covered with Bio-Gide membrane to stanch bleeding and promote wound healing followed by a suture of gingival flap. After surgery, daily surgical sites cleanse and cefazolin sodium $(20 \mathrm{mg} / \mathrm{kg} / \mathrm{day})$ administration were applied for initial 7 days to minimize the risk of postoperative infection.

\section{Fluorescent labeling design and analysis}

To monitor the osteogenic differentiation, three different fluorescent substances were introduced intravenously at defined points in time: calcein (Sigma Chemical Co.,USA) at a dose of $20 \mathrm{mg} / \mathrm{kg}$ on week 2 postoperatively; calcein blue (Sigma Chemical Co.,USA) at a dose of $20 \mathrm{mg} / \mathrm{kg}$ on week 6; and oxytetracycline (Sigma Chemical Co.,USA) at a dose of $20 \mathrm{mg} / \mathrm{kg}$ on week 10. Subsequently, the animals were sacrificed by intravenous injection of $40 \mathrm{ml}$ Thiopental (Chem-strong, China) for deep sedation and, after cessation of breathing, $40 \mathrm{ml} 15 \%$ potassium chloride for cardiac arrest. The bilateral alveolar ridges were taken and fixed in $70 \%$ alcohol solution.

The $10 \mu \mathrm{m}$-thick undecalcified ground sections were made by using a microgrinding system. A confocal microscopy (CLSM, Lsm 5 Exciter, Zeiss) was employed for 
fluorescence monitoring. The mean was calculated per osteon from the distances of the vertical line between the fluorochromic bands divided by the time interval between the applications. This value corresponded to the mineral apposition rate (MAR) in $\mu \mathrm{m} /$ day for the respective interval between marker substance applications.

\section{Toluidine blue staining}

Sections were processed for toluidine blue staining as previously described[19]. Tissue morphology were observed using an Olympus BX51 microscope equipped with a digital color camera. In each section, ten views were randomly captured for osteogenesis analysis. Image-ProPlus 6.0 was applied to determine the influence of different managements on Osseous development.

\section{Statistical analysis}

The results are expressed as means \pm standard deviation $\left(\overline{\mathrm{X}}_{ \pm} \mathrm{SD}\right)$. All data were analyzed by one-way analysis of variance (ANOVA) followed by Dunn's or Tukey's test using SPSS 18.0 (Statistical Product and Service Solutions, Inc.). A P-value of $<0.05$ was considered statistically significant.

\section{Results}

\section{Distribution of growth factors in different layers}

As shown in Figure 1, blood sample was separated into three distinct layers with clear boundaries via variable speed centrifugation: supernatant serum, CGF gel and RBC layer (from top to bottom). Furthermore, the result of immunohistochemical staining indicated that platelet and leukocyte with medium density were located between the CGF gel and RBC layer, and TGF- $\beta 1$ and VEGF were enriched in the CGF layer (Figure 2a-b). Nevertheless, a small part of growth factors was still found in the RBC layer characterized by patchy and flaky distribution. Moreover, in the RBC layer, more growth factors gathered in the area near the CGF gel. 


\section{The contents of growth factors attenuated with time course in vitro}

Generally, as the time lapse existed between preparation and application of CGF gel, the concentrations of growth factor in different time points were determined by immunohistochemical analysis to further investigate the attenuation characteristic of growth factors with time. The results of immunohistochemistry showed that the contents of growth factors in CGF gel and RBC layer seemed to be decreased over time (Figure 3). Furthermore, integrated option density was calculated by semi-quantitative analysis and presented as increasing decay rate (Table 1). However, at different time points, significant statistical differences were found in TGF- $\beta 1$ rather than VEGF (Table 2).

\section{CGF gel was viable to maintain a prolonged release of growth factors in vitro}

As previous studies reported, CGF gel has been documented as a promising carrier characterized by sustained-release function. In this study, the lifetime (up to 28 days with interval of 7 days) of CGF gel releasing growth factors was monitored by ELISA assay. The results suggested that the contents of growth factors leaking from CGF gel increased remarkably in the first 14 days, and subsequently reached plateau (Table 3 and 4). In terms of content, obviously, CGF gel could release more TGF- $\beta 1$ than VEGF, and the emission of either from CGF gel was prior to supernatant serum.

\section{Combination of CGF gel and Bio-Oss promoted osteogenesis in vivo}

To further investigate the osteo-inductive and regenerative properties of CGF gel, a beagle-based tooth-deficient socket model was established. As shown in Figure 4, new bone formation and more osteoblast islands were observed in all groups, however, obvious morphology and quantity differences were still existed. In CGF-Bio-Oss group, stable fixation between the Bio-Oss granule and cancellous bone was achieved, and granule-to-bone adhesion was less in Bio-Oss group. Besides, hypercellular granulation, increased osteoblast number, and trabecular bone were observed in CGF-Bio-Oss group. In the control group, a considerable amount of new bone, but 
with disorder internal structure, were stained clearly. Additionally, slight inflammatory cell infiltration, as well as loose and edematous soft tissue, were found at the defect area in Bio-Oss and control groups.

Furthermore, the results of fluorescent staining showed obvious space between different fluorescent bands both in CGF-Bio-Oss and Bio-Oss groups, and bands fused in the control group (Figure 5). The MAR (14-70 days) was $2.21 \pm 0.52$ $\mu \mathrm{m} /$ day in CGF-Bio-Oss group, which was higher than that in Bio-Oss $(1.43 \pm 0.41$ $\mu \mathrm{m} /$ day) and control groups (1.04 $\pm 0.26 \mu \mathrm{m} /$ day) (Table 5).

\section{Discussion}

Dental defect resulting from fractures and disease has become a medical concern, which lacked the ability for self-regeneration and requires surgical intervention. In most cases, regeneration of bone defects necessitated the use of bone grafts to replace the diseased or missing bone by natural or synthetic materials[20]. For the ideal bone graft materials, it should be balance in multiple aspects including biocompatibility, osteoinduction, resorbability or degradability, and even similar mechanical properties to those of bone at the implant site in order to develop osteogenesis and shorten the implanting period[21]. But predictable results have been still difficult to obtain due to the complex oral environment, which limited the prognosis. Previous clinical follow-up studies declared that patients received conventional treatment, comprising various types of access flaps and/or resection, were deeply troubled by probing depth reduction, hard tissue fill or even elimination of the intrabony component[22]. Furthermore, residual pockets often persisted following nonsurgical therapy or the use of access flaps, and resective techniques were associated with chronic loss of attachment and increases in soft-tissue atrophy[23]. Whatever, the consensus was that bone autograft has been identified as the criterion standard, mainly because of its powerful osteo-inductive activity[24]. Clinically, the application of autograft was, 
however, hindered by the difficulty in harvesting sufficient bone[25]. Over the years, benefiting from the development of tissue engineering, diverse novel treatment approaches involving nonsurgical techniques, as well as conservative, resective and regenerative surgical techniques have been proposed for dental defect repair/regeneration and significantly altered the way in which dentist managed dental defect, often including bone replacement graft materials, barrier membranes, various growth factors and combination of these[26, 27].

According to present available studies, combined application of two or more materials seemed to be more effective due to alveolar rebuilding and remodeling were two relative independent events but both essential for bone regeneration[28, 29]. In this study, Bio-Oss and CGF gel were employed to form a union against alveolar defect. Bio-Oss particles could function as bridge to connect distant osteoblasts, and CGF gel was responsible for providing ample growth factors to active, induce and maintain osteogenesis. Obviously, a satisfactory result has been achieved and was manifested by the vigorous osteogenesis at defect site as well as the reduction of inflammatory infiltration. Interestingly, the newly formed bone induced by combination regimen possessed regular internal structure, energetic osteoblasts and strong adhesion between the graft and bone. In the grafted site, the growing distance between distinct fluorescence labeled interfaces represented persistent osteogenesis post implantation of Bio-Oss and CGF gel. However, in terms of osteogenesis, implantation of blood clot (the control group) seemed to be prior to Bio-Oss alone which might be explained by a small amount of growth factors and fibrin residues in the clot. Whatever, these results were in accordance with previous studies that application of Bio-Oss alone might not be a rational choice for patients with dental defect because of the shortage of osteoinduction[30, 31]. Similarly, for the low abundance of growth factors, the osteogenesis mediated by blood clot remained at a relative lower level when compared to CGF gel treatment. Meanwhile, due to the lack of scaffold, the bone deposition processed without orderly orientation and could not form regular 
three-dimensional structure, hence distinct fluorescent labels appeared to be overlapped in the control group. However, the results still demonstrated that blood components contained the growth factors which were indispensable for osteogenesis.

Bio-Oss was a kind of xenograft sourced from bovine bone, and considered as an ideal alternative autogenous bone substitute and endowed with adequate new bone formation, low reabsorption rate, osteoconductive characteristics, and compensation for the natural bone resorption caused by remodeling[32]. After heating, chemical and grinding treatment, the inorganic phase of bovine bone consisted mainly of hydroxyapatite that retained the porous architecture, which could offer a scaffold for cells to proliferate and differentiate[33]. Recent studies showed some promising results comparable to autogenous bone graft. Actually, only Bio-Oss particles of appropriate size could provide sufficient contact surface for osteoblasts during the progression of bone formation and regeneration, indicating mechanical immobility was crucial for bone formation[34]. In the present study, granules $0.25 \mathrm{~mm}-1 \mathrm{~mm} / 0.5 \mathrm{~g}$ Bio-Oss was selected to mix with CGF gel at a ratio of 1:1, and the results showed that tight adhesion between has been achieved.

Recently, CGF gel has achieved acceptance as it retained a durable innate fibrin matrix, which was able to fixate platelets and growth factors and form an organic complex[35]. As an autologous material, CGF gel has been documented as a promising graft without immune responses[36]. In this study, no immune rejection occurred in any subject. Moreover, in the CGF gel treated group, the postoperative blood loss was less, and the wound healing speed was significantly faster (data not shown). Thrombin embedded in the CGF gel led to gradual polymerisation of fibrinogen into fibrin which resulted in a physiologic construction that was beneficial to wound-healing. On the other hand, the structural configuration with respect to cytokines incorporation in fibrin matrix endowed CGF gel with a prolonged lifespan and an augmented movement of cytokines in the fibrin meshes. Our data showed that the progress of growth factors releasing lasted for up to 28 days in vitro[37]. But it 
was noteworthy that the natural growth factors were liberated at the time of initial matrix remodeling, and the decay rates of different cytokines existed obvious difference. According to the immunohistochemical results, the content of TGF- $\beta 1$ was higher than VEGF, meanwhile, its decay rate was also faster than that of VEGF. Thus, in our opinion, CGF gel should be implanted within 60 min to access the best effect. Some studies reported that CGF gel also contributed to the development of micro-vasucluarisation and more functional cells migration. Certainly, all these effects were influenced by the centrifugal technique which might introduce different cytokines with different centrifugation program[38]. Optimal centrifugation was vital to harvest target growth factors. The program used in this study could separate and retain massive TGF- $\beta 1$ with a small amount of VEGF. Even though, the residual VEGF were still enough for requirement of angiogenesis.

Until now, few studies reported the combination of Bio-Oss and CGF gel in Canidae alveolar bone defect model, and our study has determined the potential of that combination as facilitator for bone regeneration. Through loaded in the Bio-Oss scaffold, CGF gel possessed the property of gradually releasing allowing clinicians to improve bone regeneration. As the mixing method used in this study was a simple physical process and autologous CGF gel, thus not much effort has been made in the ratio of CGF gel and Bio-Oss. In the further study, we hope to explore the possibility to achieve a chemically stable conjunction of CGF gel and Bio-Oss in order to access a higher loading rate and completely control the releasing progress.

\section{Conclusions}

A novel combination of CGF gel and Bio-Oss was introduced in this study, and the osteogenesis was determined in a Canidae tooth-deficient socket model. Moreover, through the observation of growth factors releasing in vitro, we found that the types of growth factors were decided by centrifugation[37]. And in the clinical application, the growth factor with the highest content, such as TGF- $\beta 1$, might influence the grafting 
time point because of higher decay rate.

\begin{abstract}
Abbreviations
CGF: concentrated growth factors; TGF- $\beta 1$ : transforming growth factor- $\beta 1$; VEGF: vascular endothelial growth factor; RBC: red blood cell; MAR: mineral apposition rate; IOD: integrated optical density; IGF: insulin-like growth factor; PRP: platelets rich plasma; PRF: platelet-rich fibrin; PBS: Phosphate Buffered Saline; SD: Standard deviation.
\end{abstract}

\title{
Acknowledgements
}

Not applicable.

\section{Authors' contributions}

The work presented here was carried out in collaboration between all authors. Peng Xue and Shao-xia Pan defined the research theme. Shao-xia Pan and Fei Chen designed methods and experiments, Fei Chen and Qiang Luo carried out the laboratory experiments, Fei Chen and Lin Wang analyzed the data, interpreted the results and wrote the paper. All authors have contributed to, seen and approved the manuscript.

\section{Funding}

This work was supported the National Natural Science Foundation of China (No. 81700968) by Peng Xue and the Scientific Research Foundation for the Returned Overseas Chinese Scholars, Ministry of Education of China (Grant No.2012-940) by Shao-xia Pan.

\section{Availability of data and materials}

The datasets generated and/ or analyzed during the current study are available from the corresponding author upon reasonable request. 


\section{Ethics approval and consent to participate}

This study protocol was reviewed and approved by the ethical board of Peking University School of Stomatology (PKUSSIRB-201519008) and the Biomedical Ethics Committee of Peking University (LA2011-060).

\section{Consent for publication}

Not applicable.

\section{Competing interests}

The authors have no conflicts of interest to disclose.

\section{References}

1. Le ADK, Enweze L, DeBaun MR, Dragoo JL. Platelet-Rich Plasma. Clin Sports Med. 2019;38(1):17-44.

2. Xiao W, Hu C, Chu C, Man Y. Autogenous Dentin Shell Grafts Versus Bone Shell Grafts for Alveolar Ridge Reconstruction: A Novel Technique with Preliminary Results of a Prospective Clinical Study. Int J Periodontics Restorative Dent. 2019;39(6):885-93.

3. Honda H, Tamai N, Naka N, Yoshikawa H, Myoui A. Bone tissue engineering with bone marrow-derived stromal cells integrated with concentrated growth factor in Rattus norvegicus calvaria defect model. Journal of artificial organs : the official journal of the Japanese Society for Artificial Organs. 2013;16(3):305-15.

4. Jensen T, Schou S, Stavropoulos A, Terheyden H, Holmstrup P. Maxillary sinus floor augmentation with Bio-Oss or Bio-Oss mixed with autogenous bone as graft in animals: a systematic review. Int J Oral Maxillofac Surg. 2012;41(1):114-20.

5. Aludden HC, Mordenfeld A, Hallman M, Dahlin C, Jensen T. Lateral ridge augmentation with Bio-Oss alone or Bio-Oss mixed with particulate autogenous bone graft: a systematic review. Int J Oral Maxillofac Surg. 2017;46(8):1030-8.

6. Park HC, Kim SG, Oh JS, You JS, Kim JS, Lim SC, et al. Early Bone Formation at a Femur Defect Using CGF and PRF Grafts in Adult Dogs: A Comparative Study. Implant Dent. 2016;25(3):387-93.

7. Durmuslar MC, Balli U, Dede FO, Misir AF, Baris E, Kurkcu M, et al. Histological Evaluation of the Effect of Concentrated Growth Factor on Bone Healing. J Craniofac Surg. 2016;27(6):1494 -7.

8. Xu Y, Qiu J, Sun Q, Yan S, Wang W, Yang P, et al. One-Year Results Evaluating the Effects of Concentrated Growth Factors on the Healing of Intrabony Defects Treated with or without Bone Substitute in Chronic Periodontitis. Medical science monitor : international medical journal of experimental and clinical research. 2019;25:4384-9.

9. Sheikh Z, Hamdan N, Ikeda Y, Grynpas M, Ganss B, Glogauer M. Natural graft tissues and 
synthetic biomaterials for periodontal and alveolar bone reconstructive applications: a review. Biomater Res. 2017;21:9.

10. Tabatabaei F, Aghamohammadi Z, Tayebi L. In vitro and in vivo effects of concentrated growth factor on cells and tissues. J Biomed Mater Res A. 2020;108(6):1338-50.

11. Rodella LF, Favero G, Boninsegna R, Buffoli B, Labanca M, Scarì G, et al. Growth factors, CD34 positive cells, and fibrin network analysis in concentrated growth factors fraction. Microscopy research and technique. 2011;74(8):772-7.

12. Masuki H, Okudera T, Watanebe T, Suzuki M, Nishiyama K, Okudera H, et al. Growth factor and pro-inflammatory cytokine contents in platelet-rich plasma (PRP), plasma rich in growth factors (PRGF), advanced platelet-rich fibrin (A-PRF), and concentrated growth factors (CGF). International journal of implant dentistry. 2016;2(1):19.

13. Takeda Y, Katsutoshi K, Matsuzaka K, Inoue T. The Effect of Concentrated Growth Factor on Rat Bone Marrow Cells In Vitro and on Calvarial Bone Healing In Vivo. Int J Oral Maxillofac Implants. 2015;30(5):1187-96.

14. Kobayashi E, Flückiger L, Fujioka-Kobayashi M, Sawada K, Sculean A, Schaller B, et al. Comparative release of growth factors from PRP, PRF, and advanced-PRF. Clinical oral investigations. 2016;20(9):2353-60.

15. Choukroun J, Diss A, Simonpieri A, Girard MO, Schoeffler C, Dohan SL, et al. Platelet-rich fibrin (PRF): a second-generation platelet concentrate. Part $\mathrm{V}$ : histologic evaluations of PRF effects on bone allograft maturation in sinus lift. Oral Surg Oral Med Oral Pathol Oral Radiol Endod. 2006;101(3):299-303.

16. Sacco P, Furlani F, De Marzo G, Marsich E, Paoletti S, Donati I. Concepts for Developing Physical Gels of Chitosan and of Chitosan Derivatives. Gels. 2018;4(3).

17. Chen J, Jiang H. A Comprehensive Review of Concentrated Growth Factors and Their Novel Applications in Facial Reconstructive and Regenerative Medicine. Aesthetic plastic surgery. 2020;44(3):1047-57.

18. Qiao J, An N, Ouyang X. Quantification of growth factors in different platelet concentrates. Platelets. 2017;28(8):774-8.

19. Bergholt NL, Lysdahl H, Lind M, Foldager CB. A Standardized Method of Applying Toluidine Blue Metachromatic Staining for Assessment of Chondrogenesis. Cartilage. 2019;10(3):370-4.

20. Costa FS, Silveira ER, Pinto GS, Nascimento GG, Thomson WM, Demarco FF. Developmental defects of enamel and dental caries in the primary dentition: A systematic review and meta-analysis. J Dent. 2017;60:1-7.

21. Louis PJ, Sittitavornwong S. Managing Bone Grafts for the Mandible. Oral Maxillofac Surg Clin North Am. 2019;31(2):317-30.

22. Berghuis G, Cosyn J, De Bruyn H, Hommez G, Dierens M, Christiaens V. A controlled study on the diagnostic accuracy of panoramic and peri-apical radiography for detecting furcation involvement. BMC Oral Health. 2021;21(1):115.

23. Hayashi C, Kinoshita A, Oda S, Mizutani K, Shirakata Y, Ishikawa I. Injectable calcium phosphate bone cement provides favorable space and a scaffold for periodontal regeneration in dogs. J Periodontol. 2006;77(6):940-6.

24. Yuce MO, Adali E, Turk G, Isik G, Gunbay T. Three-dimensional bone grafting in dental implantology using autogenous bone ring transplant: Clinical outcomes of a one-stage technique. Niger J Clin Pract. 2019;22(7):977-81. 
25. Nkenke E, Neukam FW. Autogenous bone harvesting and grafting in advanced jaw resorption: morbidity, resorption and implant survival. Eur J Oral Implantol. 2014;7 Suppl 2:S203-17.

26. Allan B, Ruan R, Landao-Bassonga E, Gillman N, Wang T, Gao J, et al. Collagen Membrane for Guided Bone Regeneration in Dental and Orthopedic Applications. Tissue Eng Part A. 2021;27(5-6):372-81.

27. Troeltzsch M, Troeltzsch M, Kauffmann P, Gruber R, Brockmeyer P, Moser N, et al. Clinical efficacy of grafting materials in alveolar ridge augmentation: A systematic review. J Craniomaxillofac Surg. 2016;44(10):1618-29.

28. Titsinides S, Agrogiannis G, Karatzas T. Bone grafting materials in dentoalveolar reconstruction: A comprehensive review. Jpn Dent Sci Rev. 2019;55(1):26-32.

29. Arab H, Shiezadeh F, Moeintaghavi A, Anbiaei N, Mohamadi S. Comparison of Two Regenerative Surgical Treatments for Peri-Implantitis Defect using Natix Alone or in Combination with Bio-Oss and Collagen Membrane. J Long Term Eff Med Implants. 2016;26(3):199-204.

30. Wong RW, Rabie AB. Effect of bio-oss collagen and collagen matrix on bone formation. Open Biomed Eng J. 2010;4:71-6.

31. Beltran V, Engelke W, Dias FJ, Leiva C, Fuentes R, Borie E. Occlusive barriers in combination with particulate Bio-Oss(R) graft: a pilot study on rabbit calvaria. Int J Clin Exp Med. 2014;7(7):1714-20.

32. Pang KM, Um IW, Kim YK, Woo JM, Kim SM, Lee JH. Autogenous demineralized dentin matrix from extracted tooth for the augmentation of alveolar bone defect: a prospective randomized clinical trial in comparison with anorganic bovine bone. Clin Oral Implants Res. 2017;28(7):809-15.

33. Xu D, Yao C, Gu M. [Clinical study of Bio-Oss powder and Bio-Oss collagen for site preservation during implantology]. Shanghai Kou Qiang Yi Xue. 2020;29(4):414-7.

34. Schmitt C, Lutz R, Doering H, Lell M, Ratky J, Schlegel KA. Bio-Oss(R) blocks combined with BMP-2 and VEGF for the regeneration of bony defects and vertical augmentation. Clin Oral Implants Res. 2013;24(4):450-60.

35. Fang D, Long Z, Hou J. Clinical Application of Concentrated Growth Factor Fibrin Combined With Bone Repair Materials in Jaw Defects. J Oral Maxillofac Surg. 2020;78(6):882-92.

36. Zhao QM, Gao J, Huang XX, Chen XP, Wang X. Concentrated Growth Factors Extracted from Blood Plasma Used to Repair Nasal Septal Mucosal Defect After Rhinoplasty. Aesthetic plastic surgery. 2020;44(2):511-6.

37. Wang L, Wan M, Li Z, Zhong N, Liang D, Ge L. A comparative study of the effects of concentrated growth factors in two different forms on osteogenesis in vitro. Mol Med Rep. 2019;20(2):1039-48.

38. Kao $\mathrm{CH}$. Use of concentrate growth factors gel or membrane in chronic wound healing: Description of 18 cases. Int Wound J. 2020;17(1):158-66. 


\section{Figures}

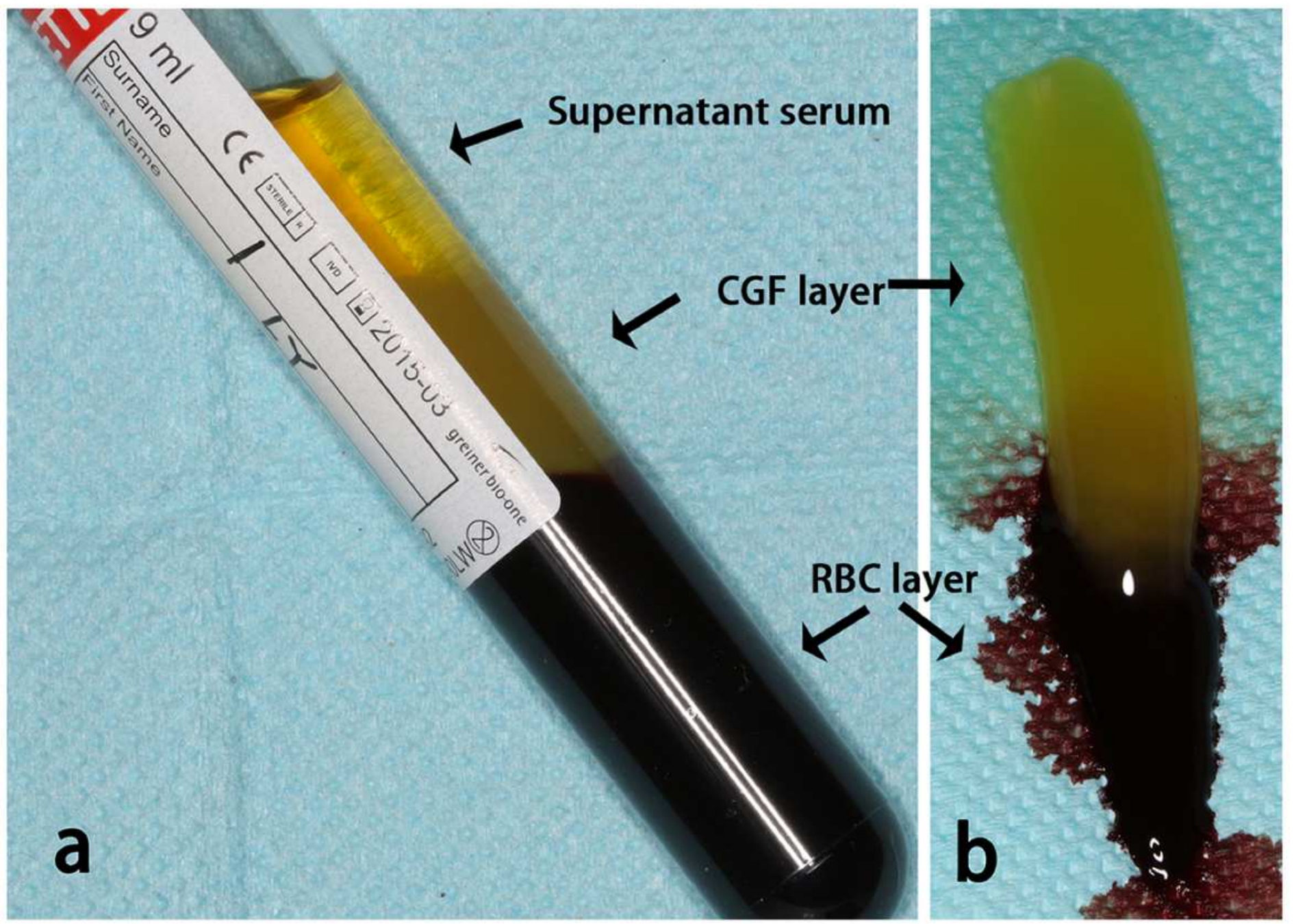

\section{Figure 1}

As shown by the arrow in the images, the blood samples were separated into three fractions via variable speed centrifugation: supernatant serum, CGF gel and RBC layers. CGF = Concentrated growth factor. $\mathrm{RBC}=$ Red blood cell. 


\section{TGF $\beta-1$}

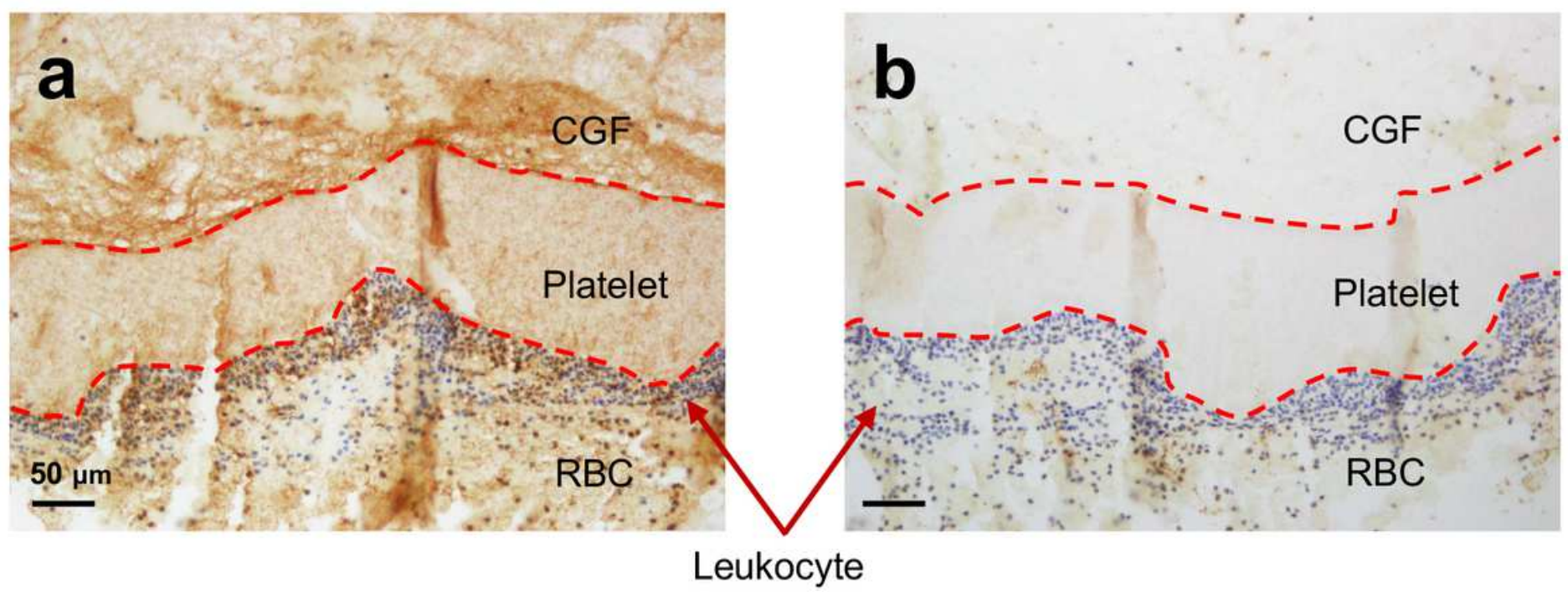

Figure 2

Evaluation of TGF- $\beta 1$ and VEGF by immunohistochemical staining. platelets and leukocytes were concentrated in abundance between CGF gel and RBC layers. Abundant TGF- $\beta 1$ and VEGF concentrated in the CGF gel along with patchy and flaky distribution in the RBC layer. However, the growth factors, found in the RBC layer, gathered in the area near the CGF gel. Scale bar $=50 \mu \mathrm{m}$. 


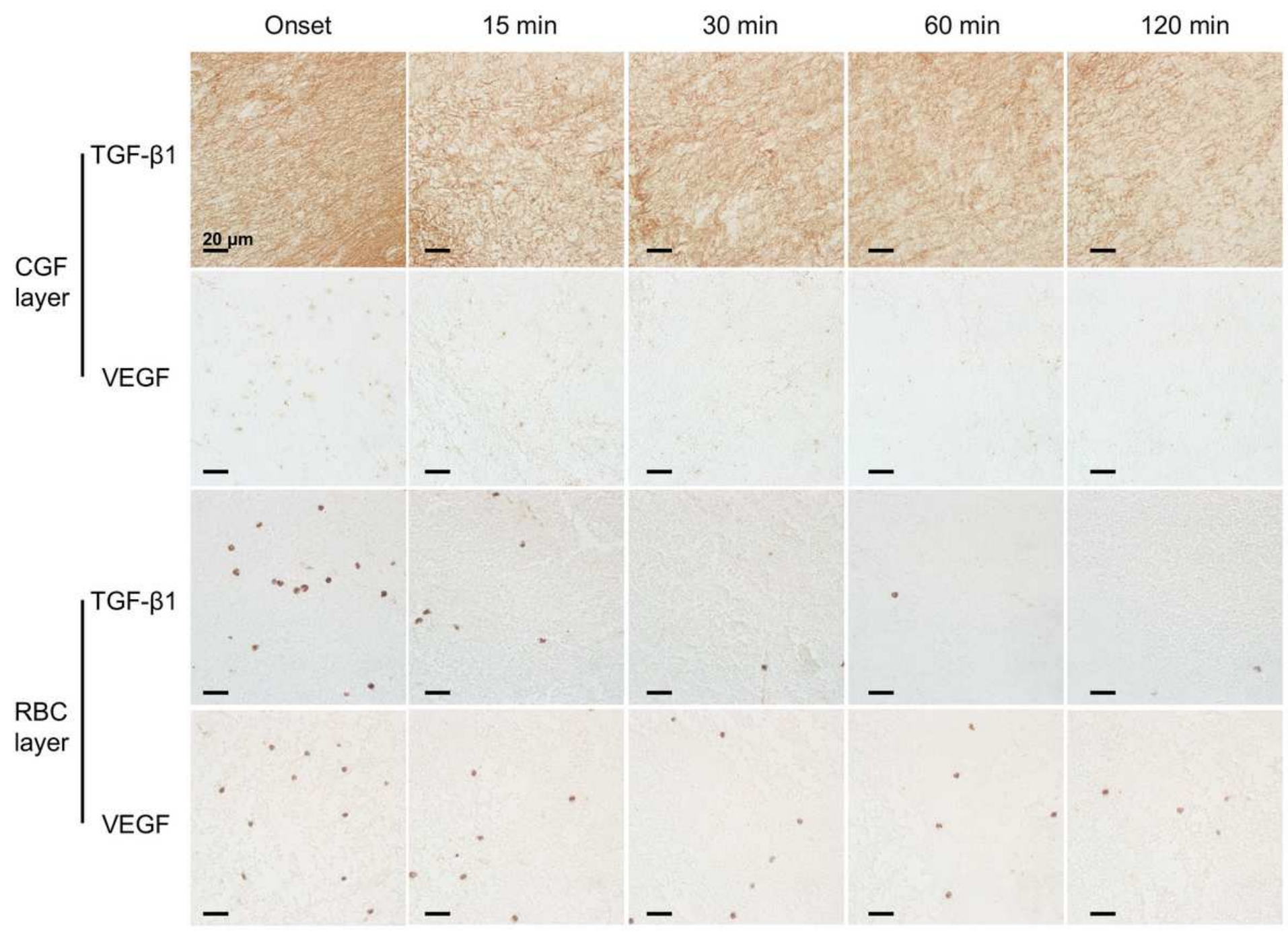

Figure 3

The content of growth factors in CGF and RBC layers decreased over the time in vitro. CGF gel and RBC layer were immunohistochemically stained by primary antibodies against TGF- $\beta 1$ and VEGF at diverse time points.

CGF-Bio-Oss

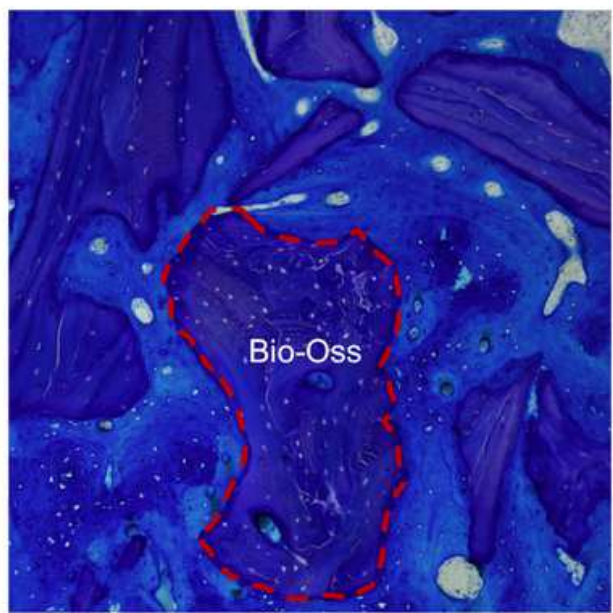

Bio-Oss

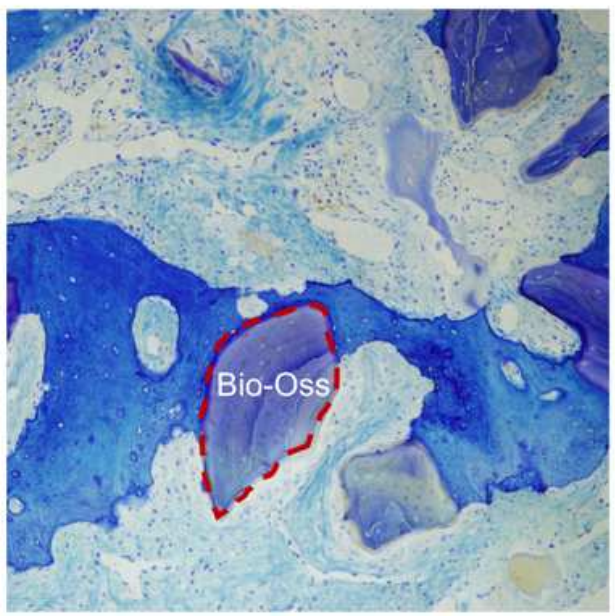

Control

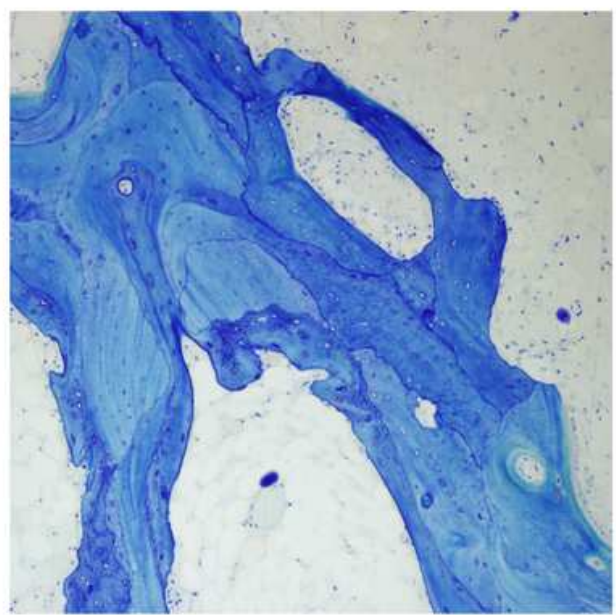




\section{Figure 4}

Representative images showing toluidine blue staining of the alveolar bone. In the CGF-Bio-Oss group, stable fixation between the Bio-Oss granule and cancellous bone was found as well as the hypercellular granulation, increased osteoblast number, and trabecular bone. Instead, less granule-to-bone adhesion was observed in Bio-Oss group, and extensive newly formed bone with disorder internal structure was found in the control group. Bio-Oss granule was highlighted through the red dotted box.

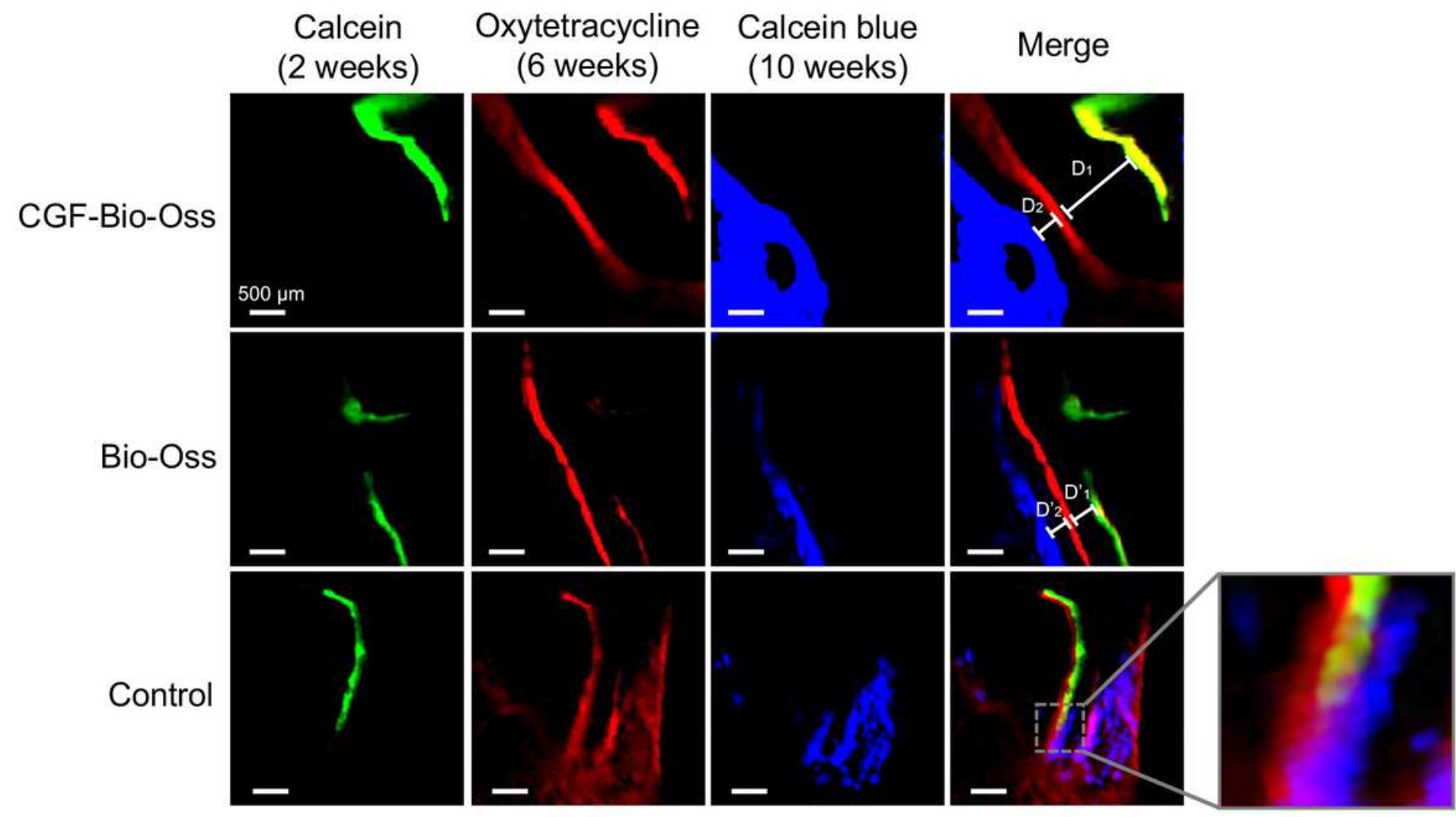

\section{Figure 5}

Calcein, calcein blue and oxytetracycline were applied to monitor the osteogenic development. Distance between different fluorescent bands was found in CGF-Bio-Oss and Bio-Oss groups, and bands fused in the control group, indicating CGF-Bio-Oss could mediate strong osteogenesis in alveolar deficient. Green: Calcein; Red: Oxytetracycline; Blue: Calcein blue. Scale bar $=500 \mu \mathrm{m}$.

\section{Supplementary Files}

This is a list of supplementary files associated with this preprint. Click to download.

- Tables.pdf 